\title{
Giant sub-THz Nonlinear Response in Superconducting Metamaterial
}

\author{
V. Savinov ${ }^{1, *}$, K. Delfanazari ${ }^{1}$, V. A. Fedotov ${ }^{1}$, and N. I. Zheludev ${ }^{1,2}$ \\ ${ }^{1}$ Optoelectronics Research Centre and Centre for Photonic Metamaterials, University of Southampton, \\ Southampton SO17 1BJ, United Kingdom \\ ${ }^{2}$ Centre for Disruptive Photonic Technologies, Nanyang Technological University, 21 Nanyang Link, 637371, Singapore \\ *vs1106@orc.soton.ac.uk
}

\begin{abstract}
We present a superconducting metamaterial with ultra-strong nonlinear response in the sub-terahertz range. A change in transmission of more than $10 \%$ has been achieved by ramping up the radiation intensity from $100 \mu \mathrm{W} / \mathrm{cm}^{2}$ to just $800 \mu \mathrm{W} / \mathrm{cm}^{2}$.

OCIS codes: (160.3918) Metamaterials; (160.4330) Nonlinear optical materials
\end{abstract}

We report on the nonlinear sub-terahertz response of the superconducting metamaterial at record-low intensity. The nonlinear response has been achieved by forcing the resonantly induced current through nano-scale constrictions, fabricated in each meta-molecule with simple UV-photolithography. An intensity-dependent change in metamaterial transmission of more than $10 \%$ has been observed by ramping up the intensity of incident radiation to just $800 \mu \mathrm{W} / \mathrm{cm}^{2}$. Such intensity corresponds to peak electric field strength of less than $1 \mathrm{~V} / \mathrm{cm}$, which is easily achieved by most terahertz sources. Our metamaterial could therefore be potentially used as a practical nonlinear component in terahertz-range devices.
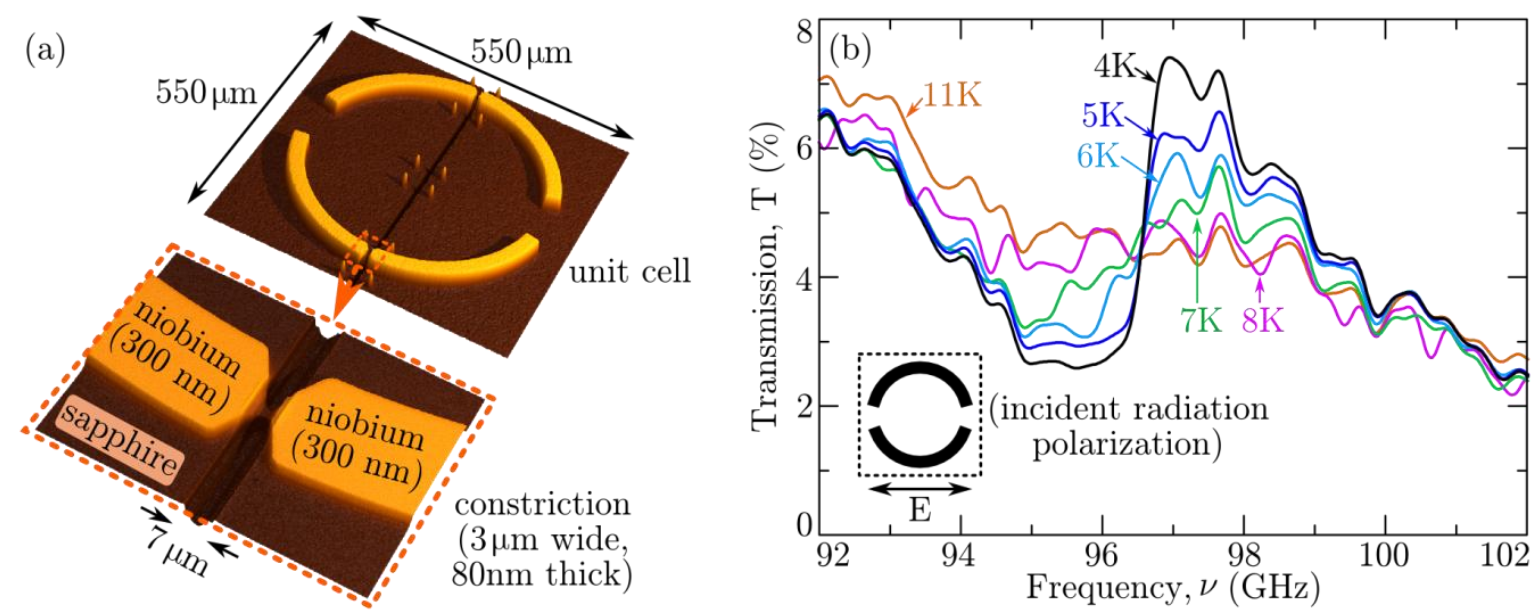

Fig. 1. Selectively-etched superconducting metamaterial. (a) Three-dimensional profile scans of the unit cell of the manufactured metamaterial (colour represents height) and of the single selectively-etched section. (b) Transmission spectra of the metamaterial at different temperatures probed with low-intensity (less than $100 \mu \mathrm{W} / \mathrm{cm}^{2}$ ) sub-THz radiation. Different curves correspond to different metamaterial temperatures. The inset shows the polarization of the incident radiation relative to the unit cell of the metamaterial.

The metamaterial, shown in Fig. 1a, was fabricated in two stages. In the first stage, a $300 \mathrm{~nm}$ thick niobium film (transition temperature, $\theta_{\mathrm{c}} \cong 9.2 \mathrm{~K}$ ), deposited on a sapphire substrate, was patterned using UVphotolithography to produce a planar array of asymmetrically-split rings. In the second stage, the same photolithographical process was used to etch groves into the metamaterial array. As a result, each asymmetrically-split ring of the metamaterial contained two narrow constrictions with cross-sections of $3 \mu \mathrm{m} \times 80 \mathrm{~nm}$. The metamaterial was placed inside a closed-cycle optical cryostat, and characterized in the spectral range $75-110 \mathrm{GHz}$ using mm-wave horn antennas and microwave network analyser.

Figure $1 \mathrm{~b}$ shows the transmission spectra of the metamaterial at different temperatures. All curves have been measured at radiation intensity below $100 \mu \mathrm{W} / \mathrm{cm}^{2}$. The metamaterial displays an asymmetric trappedmode resonance with transmission peak located at around $98 \mathrm{GHz}$. Such response, arising due to destructive interference of the radiation scattered by currents induced in the different arcs of the split rings, is typical to 
asymmetrically-split ring metamaterials. Due to reduced scattering, the currents induced in the meta-molecules, by the incident radiation, are maximized at the trapped-mode resonance. Consequently, the response of the metamaterial at this frequency becomes extremely sensitive to Joule losses, which is manifested by the collapse of the trapped-mode transmission peak when the (metamaterial) temperature is ramped from $4 \mathrm{~K}$ to $11 \mathrm{~K}$ across the superconducting transition temperature of niobium at $9.2 \mathrm{~K}$.

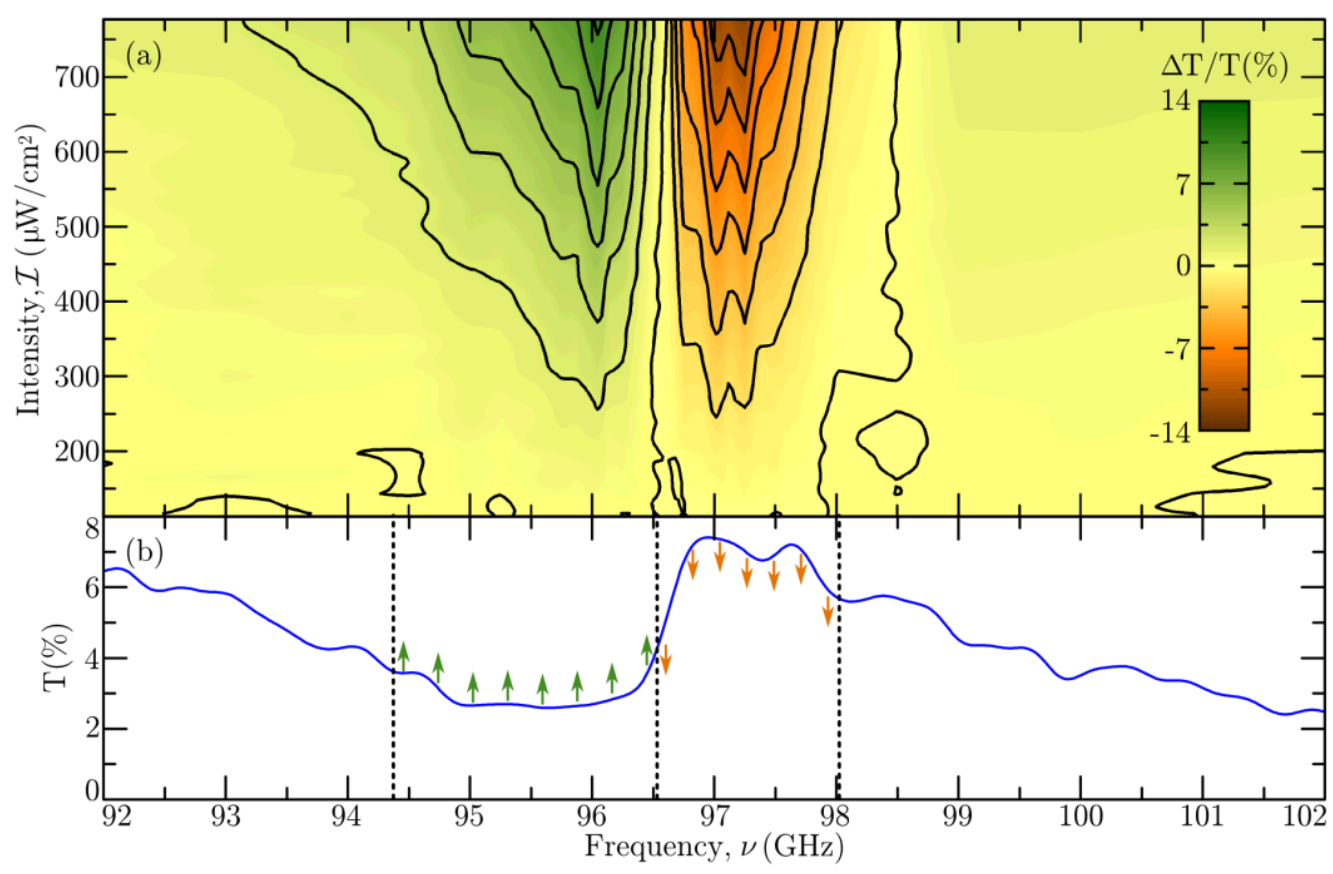

Fig. 2. Nonlinear response of the metamaterial at temperature 4K. (a) Relative change in metamaterial transmission as a function of radiation intensity and frequency. (b) Transmission of the metamaterial measured at $4 \mathrm{~K}$ with radiation intensity below $100 \mu \mathrm{W} / \mathrm{cm}^{2}$, plotted for comparison with (a). Green and orange arrows schematically depict the direction of change of the transmission spectrum in response to increasing intensity of incident radiation.

The resonant enhancement of currents induced within the split rings at the trapped mode resonance allows to reach the current densities high enough to observe the nonlinear response of the superconducting niobium in the narrow constrictions (see Fig. 1a). Figure 2a shows the relative change in metamaterial transmission as a function of radiation intensity and frequency for metamaterial kept at temperature $4 \mathrm{~K}$. One can clearly see that the transmission of the metamaterial changes as a function of intensity with largest change occurring around the trapped mode resonance of the metamaterial $\left(\Delta T / T=-12.2 \%\right.$ at intensity $777 \mu \mathrm{W} / \mathrm{cm}^{2}$ and frequency $\left.97.3 \mathrm{GHz}\right)$.

Through careful analysis of heat transfer with the constrictions it has been established that the observed nonlinearity is most likely caused by heating of the constrictions by the radiation-induced currents. However, the same analysis shows that 30 -fold increase in the amplitude of the incident electromagnetic waves would be sufficient for induced current density, at the constrictions, to reach the critical value, which would result in a much faster, potentially sub-nanosecond, nonlinear response.

In conclusion, we will present a superconducting metamaterial with ultra-strong nonlinear response in the sub-terahertz range that can be easily manufactured in two simple stages of photolithography. 